\title{
Four-Parametric Non-Linear Regression Fit of Isovolumic Relaxation in Isolated Ejecting Rat and Guinea Pig Hearts
}

\author{
Stefan F. J. LANGER \\ Institute of Physiology, Free University Berlin, Arnimallee 22, D-14195 Berlin, Federal Republic of Germany
}

\begin{abstract}
Left ventricular isovolumic pressure fall is characterized by the time constant $\tau$ obtained by fitting the exponential $\mathrm{p}(t)=p_{\infty}+\left(p_{0}-p_{\infty}\right)$ $\times \exp (-t / \tau)$ to pressure fall. It has been shown that $\tau$, calculated from the first half of pressure fall, differs considerably from that found at late relaxation in normal and pathophysiological conditions. The present study aims at testing for such differences statistically and to quantify $\tau$ changes during relaxation. Two improvements of the common regression procedure are introduced for that purpose: the use of the four-parametric regression function, $\mathrm{p}(t)=p_{\infty}+\left(p_{0}-p_{\infty}\right)$ $\times \exp \left[-t /\left(\tau_{0}+b_{\tau} t\right)\right]$, and an optimal data-dependent split of the isovolumic pressure fall interval. The residual regression errors of the methods are statistically compared in one-hundred isolated working rat and one-hundred guinea pig

hearts, additionally including a logistic regression method. Regression error is significantly reduced by introducing that $b_{\tau} . b_{\tau}$ is negative in most cases, indicating accelerated relaxation during isovolumic pressure fall, but zero and positive $b_{\tau}$ are occasionally seen. Optimal interval tripartition further improves the regression error in most cases. The statistically proved acceleration of the time constant during isovolumic relaxation justifies factor $b_{\tau}$ as a direct and continuous measure of differences between early and late relaxation. This difference between early and late isovolumic relaxation is probably caused by residually contracted myocardium at the beginning of pressure fall, and is therefore important to describe pathophysiological effects on relaxation phases. [Japanese Journal of Physiology, 50, 101-113, 2000]
\end{abstract}

Key words: myocardial contraction, diastole, ventricular relaxation, regression analysis, relaxation time constant.

Myocardial relaxation has received much attention as an important property in normal and pathologic hearts [1], especially as a sensitive indicator of beginning ("diastolic") heart failure $[2,3]$ and myocardial hypoxia [4-6]. The time constant $\tau$ of the exponential part of diastolic left ventricular pressure (LVP) fall has become a widespread index of isovolumic relaxation. The beginning of isovolumic relaxation $(t=0)$ is usually assumed at the time of peak negative pressure fall velocity, $\min \mathrm{LVdP} / \mathrm{d} t$. The time when previous end-diastolic pressure is re-encountered is chosen as the end point of isovolumic relaxation. The time course of LVP during that relaxation interval is usually fitted to the exponential:

$$
\mathrm{p}(t)=p_{\infty}+\left(p_{0}-p_{\infty}\right) \exp \frac{-t}{\tau},
$$

using non-linear least-squares regression. Three parameters, $p_{\infty}$ (asymptotic $\mathrm{p}(t)$ if $t \rightarrow \infty$ ), $p_{0}$ (estimate of LVP at $t=0$ ), and the time constant $\tau$ are subjected to regression estimation (notice that the factor of the exponential term in Eq. 1 is $p_{0}-p_{\infty}$, not $p_{0}$ as was some-

Received on June 21, 1999; accepted on December 8, 1999

Correspondence should be addressed to: Stefan F. J. Langer, Institute of Physiology, Free University Berlin, Arnimallee 22, D-14195 Berlin, Federal Republic of Germany. Tel: +49-30-8445-1649, Fax: +49-30-8445-1602, E-mail: langer@mail.grumed.fu-berlin.de

Abbreviations (refer also to Table 1): LVP, empirical left ventricular pressure curve; min LVdP/dt, peak negative LVP fall velocity; $p$, diverse regression functions to fit isovolumic LVP fall; $p_{0}$, theoretical initial pressure, $p_{0}=p(0) ; p_{\infty}$, theoretical pressure asymptote, $p_{\infty}=\lim _{t \rightarrow \infty} p(t) ; \tau$, exponential time constant of pressure fall in model Eq. 2, generally: a time-dependent function defined by Eq. $5 ; \tau_{0}$, initial $\tau$ at $t=0 ; b_{\tau}$, steepness of $\tau$ in model Eq. 2; $\tau^{*}$, central time constant estimated from Eq. 2 by $\tau^{*}=\tau_{0}+b_{\tau} t^{*}$, wherein $t^{*}$ is the central point (i.e., half the length) of the time interval of isovolumic pressure fall; $\tau_{\infty}$, asymptotic $\tau$ at $t \rightarrow \infty$ in model Eq. 3 ; $\mathrm{BI}$, interbeat interval; $C_{90}$, central $90 \%$ range of a set of data (i.e., the shortest possible interval which covers at least $90 \%$ of the data). 


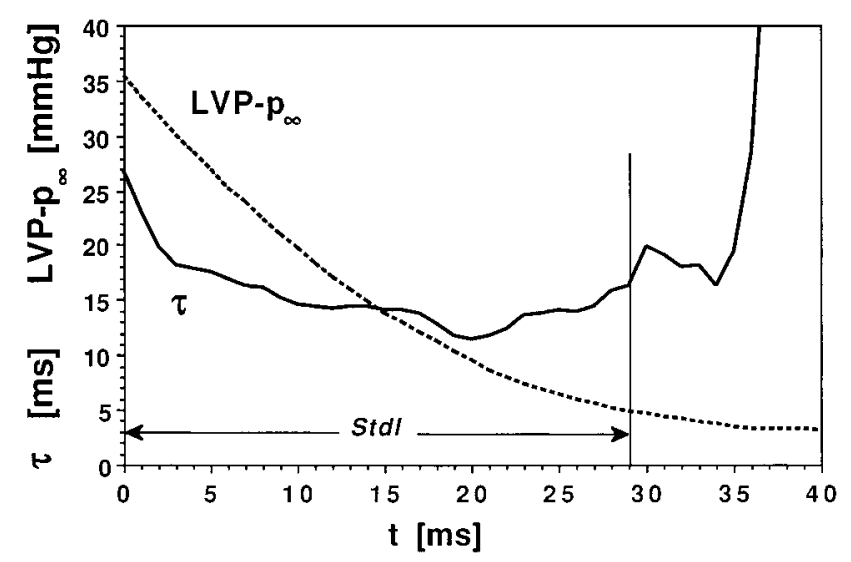

Fig. 1. Left ventricular pressure curve, adjusted to zero asymptote (LVP $\left.-p_{\infty}\right)$, during isovolumic relaxation of an isolated working guinea pig heart at $37^{\circ} \mathrm{C}$, mean aortic pressure $60 \mathrm{mmHg}$, beat interval $232 \mathrm{~ms}$, cardiac output $40 \mathrm{ml} \mathrm{min}^{-1}$. $p_{\infty}$ was previously calculated by fitting Eq. 1 to LVP data and then subtracting from the empirical LVP data. Equation 1 solves for the differential equation $\mathrm{dp}(t) / \mathrm{d} t=-\tau^{-1}\left[\mathrm{p}(t)-p_{\infty}\right]$, therefore an empirical time-dependent function of the pressure fall time constant is obtained by $\tau(t)=-\left[\operatorname{LVP}(t)-p_{\infty}\right][\operatorname{LVdP}(t) / d t]^{-1}$ and is also shown in the panel. $t=0$ is the time of peak negative pressure fall velocity, min $\mathrm{LVdP} / \mathrm{d} t$; the standard relaxation interval, Stdl, is ending at re-encountered previous end-diastolic pressure. The $U$-shaped $\tau$ curve is typical and has already been seen in other hearts [12]. Notice that $\tau(t)$ is unreliable by severe round-off errors at high $t$ because the divisor becomes very small.

times miswritten $[1,5,7]) . p_{\infty}$ is often preset to zero using a two-parametric linear regression of logarithmized pressure data, $\ln \mathrm{p}(t)=\ln p_{0}-t / \tau[2,4,8]$, but Eq. 1 improves the fit significantly [9-11]. It was already suggested from two-dimensional regression that the time constant $\tau$ may be smaller at late relaxation, indicating acceleration of pressure fall [4]; but this may not indicate true differences in $\tau$ since the assumption $p_{\infty}=0$ is not justified [10].

Figure 1 demonstrates that the situation is more complex. Plotting empirical $\tau$ as a time-dependent function reveals that only its middle region seems suitable to be fitted to a linear function. The U-shape of $\tau$ and its slight decrease during the central part of the relaxation interval are both typical. $\tau(t)$ is considerably higher at small $t$, and especially at high $t$.

The aim of the present study was to check statistically for a possible continuous change of $\tau$ during isovolumic relaxation in isolated working left ventricles of rat and guinea pig, and to make use of a positive result to propose an improved regression function for isovolumic pressure fall. Equation 1 was extended, for this purpose, to a four-parametric regression function,

$$
\mathrm{p}(t)=p_{\infty}+\left(p_{0}-p_{\infty}\right) \exp \frac{-t}{\tau_{0}+b_{\tau} t},
$$

to cover variations of $\tau$. This extension was tested against Eq. 1 for significant improvement of fit in onehundred rat and one-hundred guinea pig hearts working at standardized conditions. A data-dependent tripartition algorithm [12] was then additionally applied to exclude the marginal LVP which do not properly fit to the regression function Eq. 2 (refer to Fig. 1).

The (three-parametric) logistic model,

$$
\mathrm{p}(t)=p_{\infty}+\frac{2\left(p_{0}-p_{\infty}\right)}{1+\exp \left(t / \tau_{\infty}\right)},
$$

where $\tau_{\infty}$ denotes the asymptotic ("late") time constant of pressure fall (see APPENDIX A), is also included in the comparison of goodness-of-fit because Matsubara et al. have demonstrated that this model is superior to the three-parametric exponential Eq. 1 [13].

\section{METHODS}

1) Preparation. The experimental setup was recently described in detail [9]. The hearts of onehundred Sprague-Dawley rats and one-hundred guinea pigs were rapidly excised under full anesthesia (according to the German Animal Protection Act) and mounted to an artificial circulation apparatus containing modified Krebs-Henseleit bicarbonate buffer ( $10 \mathrm{mmol} l^{-1}$ glucose and $2 \mathrm{mmol} l^{-1}$ sodium pyruvate added, $95 \% \mathrm{O}_{2}, 5 \% \mathrm{CO}_{2}, \mathrm{pH} 7.4,37^{\circ} \mathrm{C}$ ). The left atrium was fed by an adjustable roller pump. Caval veins were ligated, and the venous effluent was collected via the pulmonary artery and monitored. Aortic pressure was regulated by an adjustable hydraulic resistor and measured by a pressure transducer. Aortic output passed a windkessel (13 $\mathrm{ml}$ air buffer, providing elasticity) and was measured by an electromagnetic flow probe. Cardiac output was assessed by adding aortic and coronary flow. High fidelity LVP curves were obtained by a subminiature catheter pressure transducer located in the left ventricular cavity (via aortic valves). The data were on-line digitized to 12-bit integers, approximately $0.075 \mathrm{mmHg}$ per bin, at a sampling rate of $1 \mathrm{kHz}$ and stored by a personal computer.

The isolated heart was allowed to stabilize for $15 \mathrm{~min}$ at the aortic pressure of $75 \mathrm{mmHg}$ (rat) and $60 \mathrm{mmHg}$ (guinea pig) and $40 \mathrm{ml} \mathrm{min}^{-1}$ cardiac output. A four-second LVP interval was then recorded at these conditions and used as original data for the sub- 

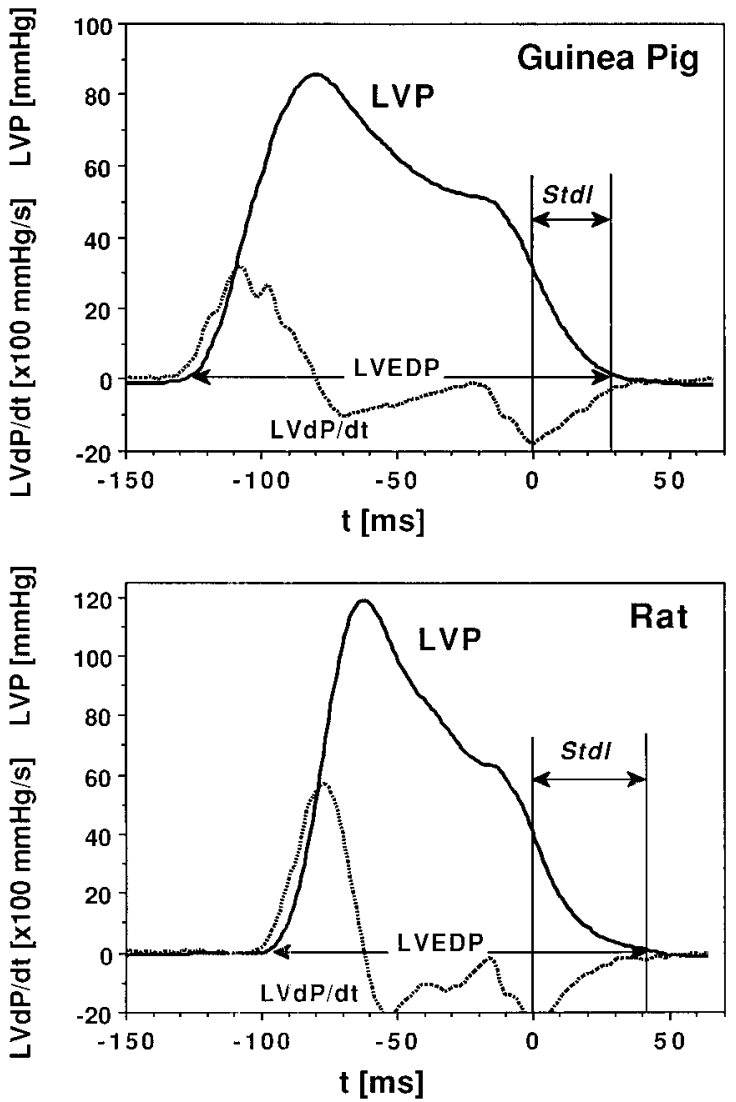

Fig. 2. Left ventricular pressure curves (LVP) and their derivatives $(\mathrm{LVdP} / \mathrm{d} t)$ of isolated working hearts from guinea pig (mean aortic pressure $60 \mathrm{mmHg}$, beat interval $232 \mathrm{~ms}$, same specimen as in Fig. 1) and rat (mean aortic pressure $75 \mathrm{mmHg}$, beat interval $230 \mathrm{~ms}$ ) at $37^{\circ} \mathbf{C}$, cardiac output $\mathbf{4 0 ~ m / ~ m i n}{ }^{-1}$. $t=0$ is the time of $\min$ $\mathrm{LVdP} / \mathrm{d} t$ (maximal velocity of pressure fall). Stdl is the standard relaxation interval ending at re-encountered previous end-diastolic pressure (LVEDP).

sequent analysis. Representative single-beat LVP curves are shown in Fig. 2.

The preparations were performed by the author and other members of the working group. Different experiments not mentioned here followed the initial data sampling; thus no animals had to be sacrified only for the purpose of the present investigation.

2) Data processing. The LVP records were partitioned into individual beat intervals by self-developed software. Derivative function $\mathrm{LVdP} / \mathrm{d} t$ was numerically calculated as steepness of the linear regression line through each of five adjacent pressure data points. End of diastole was determined at the rapid upstroke of $\mathrm{LVdP} / \mathrm{d} t$. The time of $\min \mathrm{LVdP} / \mathrm{d} t$ was identified in each beat and set to zero for convenience. The relaxation phases of all beats from the four-second sampling interval were pooled by adjusting the abscissa to zero. Two different interval lengths were selected: a standard interval, StdI, and an extended in- terval, ExtI (see Table 1a for definition).

StdI was subjected to four fitting protocols which are described in Table 1b. Equation 1 was fitted with an iterative analytic method which is described in detail elsewhere [14]; to fit Eqs. 2 and 3, an initial recursive search for global regression error minima was performed (see APPENDIX B), and the found minima were subsequently improved using the simplex algorithm according to (Press et al. [15], pp. 289-293).

ExtI was partitioned into three subintervals which were seperately fitted to Eq. 2. All possible tripartitions were taken into account as described elsewhere [12]. First and last subintervals were discarded and only the central interval, ExtC, was further considered as a reliable reference of the undistorted isovolumic relaxation phase. The above-mentioned four fitting protocols were also applied to ExtC (see Table 1b).

3) Statistical hypotheses and tests. Sums of squared residual errors were calculated for each heart and regression method. Appropriate degrees of freedom were determined as difference between the respective sample sizes (i.e., the sum of the milliseconds-length of all relaxation intervals from the 4-s sampling interval, typical values for the standard interval StdI: 480-650) and the number of parameters estimated; the splitting points in the tripartition method on ExtI were treated as two additional parameters because they were also estimated from the data. The following zero hypotheses (assuming equal error variance in the respective pair of methods) were statistically tested in each heart separately.

StdI:

S.1) StdI3 vs. StdIL C.1) ExtC3 vs. ExtCL

S.2) StdIL vs. StdI4 C.2) ExtCL vs. ExtC4

S.3) StdI3 vs. StdI3h C.3) $\operatorname{ExtC} 3$ vs. ExtC3h

S.4) StdI3h vs. StdI4 C.4) ExtC3h vs. ExtC4

Unilateral alternative hypotheses were adopted, stating that the respective second model in the above list yields significantly less regression error, because the opposite cases are of no interest. The goodness-offit of selected pairs of methods was compared by calculating Fisher's $F$ statistic,

$$
F=\frac{\left(\mathrm{SS}_{1}-\mathrm{SS}_{2}\right) d_{2}}{\mathrm{SS}_{2}\left(d_{1}-d_{2}\right)}
$$

wherein SS refers to the sum of squares, $d$ refers to the degrees of freedom, and subscript 1 denotes the supposed inferior fit [16]. Individual error probabilities $p$ were calculated from the $F$ distribution (using $d_{1}-d_{2}$ and $d_{2}$ degrees of freedom) by means of the incomplete beta function (Press et al. [15], p. 190). To obtain a global decision, the $p$ values (among one 
Table 1. Definitions of regression data intervals and regression models used in the study.

a) LVP intervals used for regression

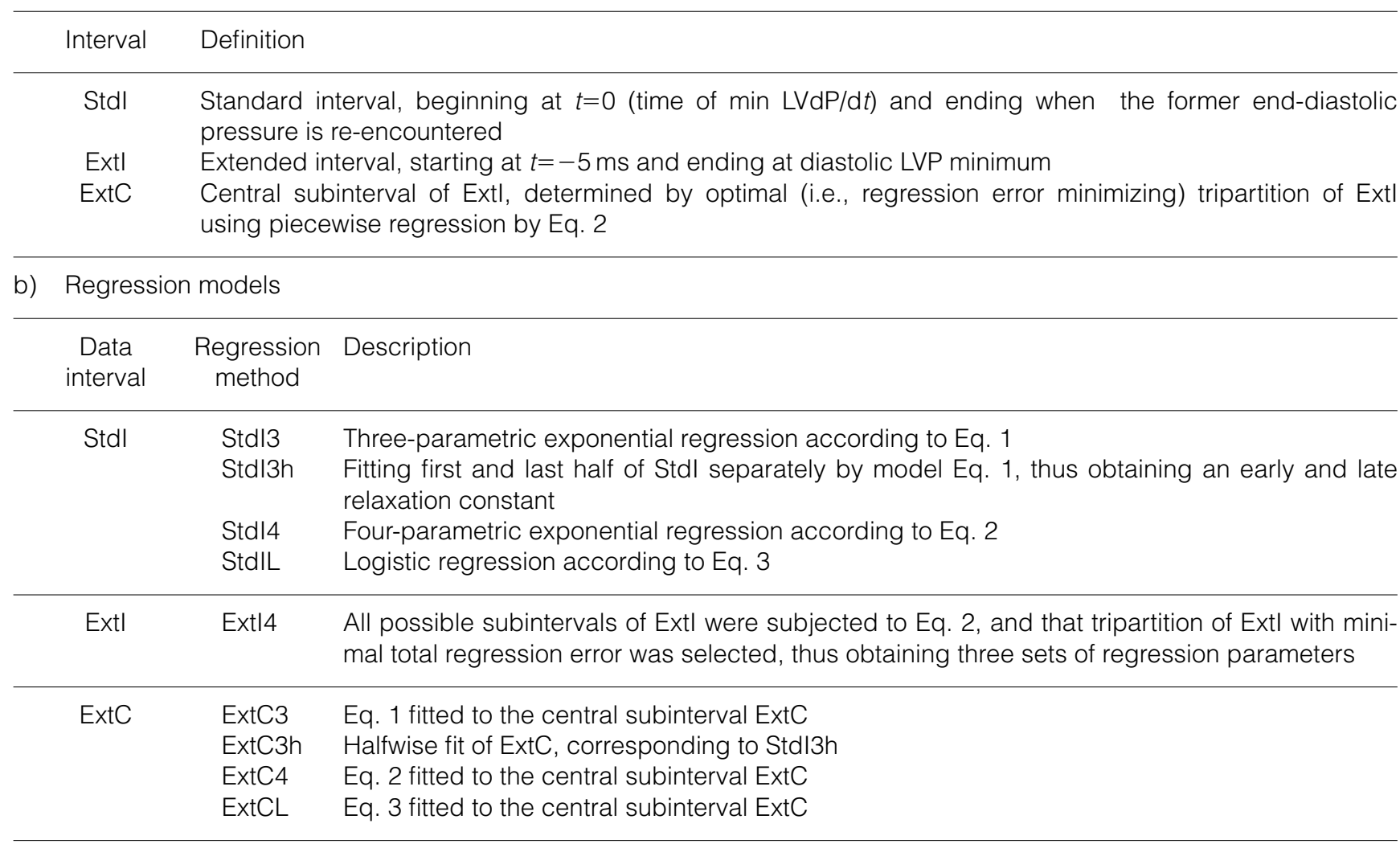

Table 2. Characteristic data (central $90 \%$ range and median) of one-hundred isolated ejecting rat and one-hundred guinea pig hearts under control conditions: $37^{\circ} \mathrm{C}$, aortic pressure $75 \mathrm{mmHg}$ (rat) resp. $60 \mathrm{mmHg}$ (guinea pig), and cardiac output $40 \mathrm{ml} \mathrm{min}^{-1}$.

\begin{tabular}{|c|c|c|c|c|c|c|}
\hline \multirow{2}{*}{$\frac{\text { Variable }}{\text { Left ventr. mass* }^{\star}(\mathrm{mg})}$} & \multicolumn{3}{|c|}{ Rat } & \multicolumn{3}{|c|}{ Guinea pig } \\
\hline & 661 & 779 & 884 & 645 & 837 & 1,065 \\
\hline Beat interval (ms) & 161 & 190 & 218 & 210 & 231 & 254 \\
\hline $\operatorname{LVEDP}(\mathrm{mmHg})$ & 0.9 & 3.1 & 5.1 & 0.0 & 4.0 & 5.3 \\
\hline $\max \operatorname{LVP}(\mathrm{mmHg})$ & 99.7 & 119.9 & 129.5 & 75.8 & 84.3 & 97.2 \\
\hline $\max \mathrm{LVdP} / \mathrm{d} t\left(\mathrm{mmHg} \mathrm{s}^{-1}\right)$ & 3,946 & 4,833 & 5,976 & 2,068 & 2,718 & 3,272 \\
\hline $\min \mathrm{LVdP} / \mathrm{d} t\left(-\mathrm{mmHg} \mathrm{s}^{-1}\right)$ & 1,623 & 2,367 & 2,872 & 1,205 & 1,740 & 2,243 \\
\hline Coronary flow $\left(\mathrm{ml} \mathrm{min}{ }^{-1}\right)$ & 12.6 & 16.0 & 19.5 & 8.3 & 13.7 & 20.4 \\
\hline
\end{tabular}

*Left ventricle incl. intraventricular septum, wet.

species) were converted to the appropriate unilateral abscissa $z$ of the standard normal distribution, and agglutinated $Z=\left(\sum_{i=1}^{n} z_{i}\right) / \sqrt{n}$ was calculated, wherein $n=100$ is the number of hearts for each species. The agglutinated (global) error probability $P$ was finally determined by reconverting $Z$. Individual significance is assumed if $P<0.001$. All significant results found among the eight comparisons are therefore simultaneously confident at the error probability of 0.01 .

\section{RESULTS}

The apparent basal data of the hearts are summarized in Table 2.

\section{Parameters of relaxation}

Table 3 displays the exponential relaxation parameters $\tau_{0}\left(\tau\right.$, respectively) and $b_{\tau}$, and the observed standard errors of regression. Data are given as median accompanied with the limits of the shortest interval which covers at least $90 \%$ of the sample (i.e., the central 90\% range, $C_{90}$ ). The logistic relaxation (Eq. 3) has not provided substantial information different from that obtained by Eq. 1. Calculating the central logistic time "constant" $\tau^{*}=\tau\left(t^{*}\right)$ from Eq. 6 (APPENDIX $\mathrm{A} ; t^{*}$ is half the length of the individual regres- 
Table 3. Comparison of exponential methods to fit left ventricular pressure during relaxation in one-hundred rat and one-hundred guinea pig hearts at $37^{\circ} \mathrm{C}$, aortic pressure $75 \mathrm{mmHg}$ (rat) resp. $60 \mathrm{mmHg}$ (guinea pig), cardiac output $40 \mathrm{ml} \mathrm{min}^{-1}$.

\begin{tabular}{|c|c|c|c|c|c|c|c|c|c|c|c|}
\hline \multirow{3}{*}{$\begin{array}{l}\text { Fitting } \\
\text { method } \\
\text { Stdl3 }\end{array}$} & \multicolumn{6}{|c|}{ Rat } & \multicolumn{5}{|c|}{ Guinea pig } \\
\hline & \multicolumn{3}{|c|}{$\tau$ resp. $\tau_{0}^{*}(\mathrm{~ms})$} & \multirow{2}{*}{\multicolumn{2}{|c|}{$\begin{array}{l}b_{\tau} \\
-\end{array}$}} & \multirow{2}{*}{$\frac{\operatorname{SER}(\mathrm{mmHg})}{0.68}$} & \multicolumn{3}{|c|}{$\tau$ resp. $\tau_{0}^{*}(\mathrm{~ms})$} & \multirow{2}{*}{$b_{\tau}$} & \multirow{2}{*}{$\frac{\operatorname{SER}(\mathrm{mmHg})}{0.50}$} \\
\hline & 11.1 & 16.7 & 26.4 & & & & 7.1 & 20.6 & 32.2 & & \\
\hline Stdl3h & & - & & & - & 0.49 & & - & & - & 0.44 \\
\hline 1st half & 6.3 & 19.9 & 59.5 & & - & 0.64 & $<0$ & 21.7 & $>200$ & - & 0.53 \\
\hline 2nd half & $<0$ & 11.7 & $>200$ & & - & 0.25 & $<0$ & 13.5 & $>200$ & - & 0.25 \\
\hline Stdl4 & 11.3 & 16.7 & 25.0 & -0.52 & -0.170 .13 & 0.51 & 10.4 & 21.8 & 37.3 & $-0.50-0.190 .18$ & 0.41 \\
\hline Ext|4 & & - & & & - & 0.44 & & - & & - & 0.35 \\
\hline ExtC4 & 0.4 & 17.7 & 38.3 & -0.59 & -0.230 .33 & 0.40 & 12.8 & 23.9 & 44.8 & $-0.63-0.310 .32$ & 0.32 \\
\hline ExtC3 & 8.4 & 14.6 & 24.9 & & - & 0.51 & 6.5 & 18.8 & 30.6 & - & 0.43 \\
\hline ExtC3h & & - & & & - & 0.44 & & - & & - & 0.37 \\
\hline 1st part & 2.7 & 18.3 & 35.6 & & - & 0.53 & 5.6 & 25.7 & 47.6 & - & 0.41 \\
\hline 2nd part & 5.2 & 13.5 & $>200$ & & - & 0.28 & 4.8 & 15.7 & $>200$ & - & 0.19 \\
\hline
\end{tabular}

Data are given as distribution (central $90 \%$ range and median) of $\tau, b_{\tau}$, and standard error of regression (SER).

${ }^{\star} \tau$ in three-parametric fits (Eq. 1), $\tau_{0}$ in four-parametric fits (Eq. 2).

sion interval StdI) and comparing it with the exponential time constant $\tau$ (Eq. 1) yielded correlation coefficients of 0.9797 (rat) and 0.9935 (guinea pig). The linear regressions are: $\tau^{*}=(0.585 \pm 0.012) \tau+$ $(1.35 \pm 0.22) \mathrm{ms}$ in rat hearts and $\tau^{*}=(0.579 \pm 0.007) \tau$ $+(1.71 \pm 0.16) \mathrm{ms}$ in guinea pig hearts with a standard error of regression less than $0.63 \mathrm{mmHg}$ in both species. The ratio $\tau^{*} / \tau$ was 0.658 and 0.644 in rat and guinea pig hearts, respectively. Notice that all of these results are valid only for the standard working conditions described before; an example of different behaviour of exponential and logistic time constants is discussed below (Fig. 5).

All LVP intervals could be successfully fitted by all methods. Careful initial search for global error minima (APPENDIX B) was found to be essential for success in the four-dimensional model. Standard regression errors, usually less than $1 \mathrm{mmHg}$, are better than approximately $1 \%$ of the pressure maximum. Figure 3 shows a representative LVP interval (ExtI) with threefold piecewise four-dimensional data fit and a threedimensional fit for the standard relaxation interval, StdI. No systematic error can be seen among the fourdimensional fit, whereas the three-dimensional model tends to underestimate late pressure values.

Four-dimensional regression reveals a diminishing rather than constant $\tau$ during isovolumic relaxation in most hearts; that is, accelerating relaxation, but the opposite effect is also seen in some hearts. This applies both to standard relaxation interval StdI and the central part ExtC of the extended fitting interval. The piecewise regressions, StdI3h and ExtC3h, also pre- sent a smaller late relaxation constant in most cases.

Optimal tripartition of ExtI results in (median and $C_{90}$ interval): $\quad 5.5-18.2-32.8 \%$ (rat) resp. $6.8-17.6-24.7 \%$ (guinea pig) preliminary interval, $32.1-48.3-62.3 \%$ (rat) resp. 40.0-52.2-66.7\% (guinea pig) central interval $(=$ ExtC), and 19.6-34.5-40.0\% (rat) resp. 19.6-31.7-39.7\% (guinea pig) terminal subinterval. The median quotient ExtC/StdI is $72.4 \%$ (rat) resp. $77.3 \%$ (guinea pig).

$p_{\infty}$ is estimated to be negative in most cases of three-dimensional fits in guinea pigs, median for StdI3: $-2.1 \mathrm{mmHg}$, median for ExtC3: $-1.4 \mathrm{mmHg}$. Conversely, four-dimensional fits yield positive $p_{\infty}$, $1.3 \mathrm{mmHg}$ (StdI4) and $2.3 \mathrm{mmHg}$ (ExtC4). In rat hearts, $p_{\infty}$ values tended to be zero.

All calculations were repeated on a beat-by-beat basis to obtain interbeat variability of the respective fitted parameters among the four-second intervals (10 to 18 beats). Median standard errors of the mean and coefficients of variations are presented in Table 4. $p_{\infty}$ and $b_{\tau}$ present considerable interbeat variability. Variability is higher in Eq. 2 because an additional parameter is to be estimated in this equation as compared with the other models.

\section{Statistical comparison of relaxation models}

Table 5 presents the results of methodical comparisons performed in one-hundred rat and one-hundred guinea pig hearts. All comparisons reveal differences in goodness-of-fit with error probability $P$ less than 0.001 except for the halfwise three-parametric fit of StdI against four-parametric fit in rat hearts. Conse- 

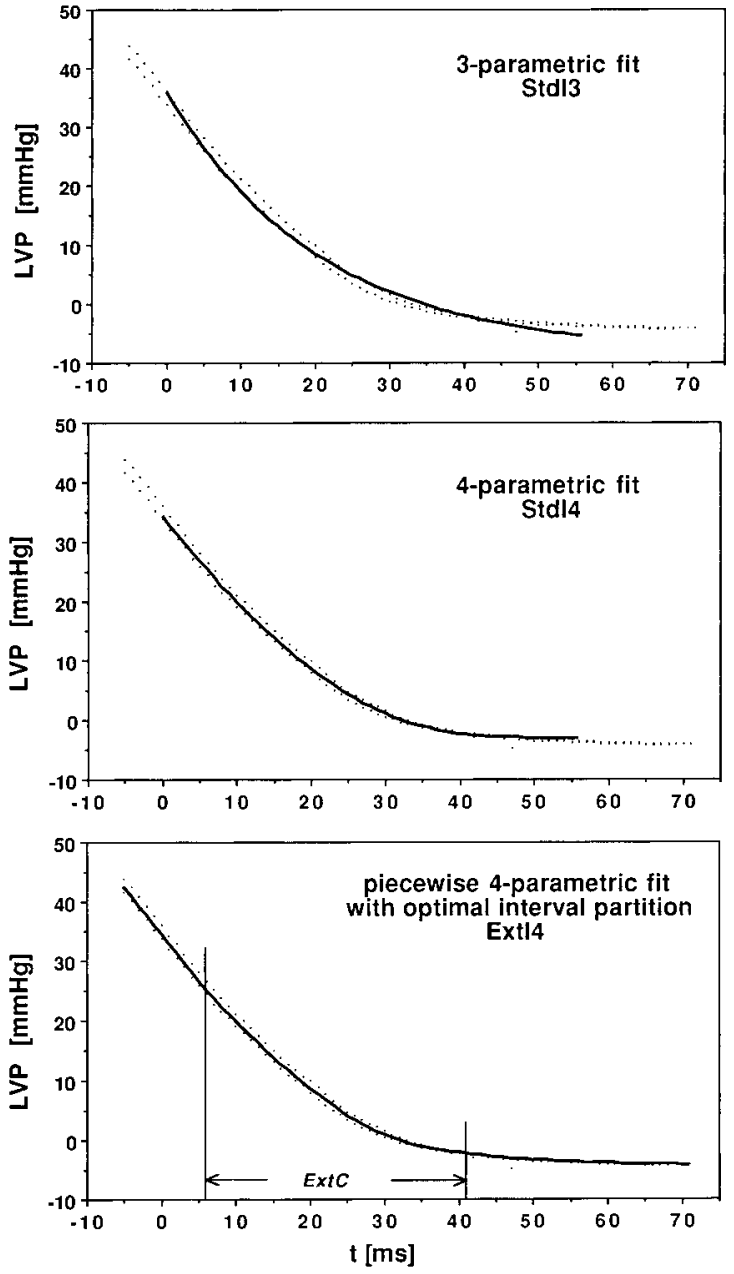

Fig. 3. Comparison of three models to fit isovolumic pressure fall in an isolated working guinea pig heart. Two dots at each abscissa mark the left ventricular pressure (LVP) range of 14 consecutive beats. Controlled condition: $37^{\circ} \mathrm{C}$, mean aortic pressure $60 \mathrm{mmHg}$, cardiac output $40 \mathrm{ml} \mathrm{min}{ }^{-1} . t=0$ is the time of peak negative pressure fall velocity, $\min \mathrm{LVdP} / \mathrm{d}$ t; former end-diastolic pressure is reached at $t=56 \mathrm{~ms}$. Three- and four-parametric fits apply to the standard interval Stdl, $t=0$ to $56 \mathrm{~ms}$. Four-parametric fit with optimal interval partition applies to the extended interval Extl, $t=-5$ to $71 \mathrm{~ms}$ (i.e., time of LVP minimum); the central subinterval of the piecewise fit (ExtC) was determined by optimal interval partition and is indicated in the bottom panel. Three-parametric fit, method Stdl3 according to Eq. 1, presents with remarkable systematic deviation from the empirical LVP (upper panel). Four-parametric fit, method Stdl4, Eq. 2, is better adapted to the data (middle panel). Three piecewise fits to the extended interval by Eq. 2 yield a reasonable fit in the full interval (bottom panel).

quently, all of these differences are simultaneously significant with an error probability of less than 0.01 .

Zero hypotheses ("equal variance") are tested against unilateral alternatives, stating that the method mentioned second is superior over the one mentioned first. The comparisons are therefore transitive. Especially, entries S.1+S.2 (E.1+E.2, respectively) from
Table 4. Standard error of the mean (SEM) and coefficient of variation ( $V$ ) of fitted parameters of ten to eighteen consecutive beats in isolated ejecting hearts.

\begin{tabular}{|c|c|c|c|c|c|}
\hline \multirow{2}{*}{ Method } & \multirow{2}{*}{ Parameter } & \multicolumn{2}{|c|}{ Rat } & \multicolumn{2}{|c|}{ Guinea pig } \\
\hline & & SEM & $V(\%)$ & SEM & $V(\%)$ \\
\hline \multirow[t]{3}{*}{ Stdl3 } & $p_{0}(\mathrm{mmHg})$ & 0.261 & 1.82 & 0.224 & 1.60 \\
\hline & $p_{\infty}(\mathrm{mmHg})$ & 0.122 & 8.90 & 0.164 & 10.54 \\
\hline & $\tau(\mathrm{ms})$ & 0.080 & 2.11 & 0.148 & 2.52 \\
\hline \multirow[t]{4}{*}{ Stdl4 } & $p_{0}(\mathrm{mmHg})$ & 0.255 & 1.75 & 0.215 & 1.55 \\
\hline & $p_{\infty}(\mathrm{mmHg})$ & 0.091 & 9.54 & 0.200 & 35.21 \\
\hline & $\tau_{0}(\mathrm{~ms})$ & 0.102 & 2.28 & 0.106 & 2.19 \\
\hline & $b_{\tau}$ & 0.004 & 11.83 & 0.007 & 17.14 \\
\hline \multirow[t]{3}{*}{ ExtC3 } & $p_{0}(\mathrm{mmHg})$ & 0.272 & 1.90 & 0.212 & 1.56 \\
\hline & $p_{\infty}(\mathrm{mmHg})$ & 0.092 & 9.02 & 0.094 & 7.50 \\
\hline & $\tau(\mathrm{ms})$ & 0.088 & 2.52 & 0.093 & 2.11 \\
\hline \multirow[t]{4}{*}{ ExtC4 } & $p_{0}(\mathrm{mmHg})$ & 0.347 & 2.55 & 0.319 & 2.55 \\
\hline & $p_{\infty}(\mathrm{mmHg})$ & 0.718 & 64.27 & 0.264 & 28.37 \\
\hline & $\tau_{0}(\mathrm{~ms})$ & 0.268 & 7.35 & 0.287 & 4.72 \\
\hline & $b_{\tau}$ & 0.024 & 49.63 & 0.015 & 31.14 \\
\hline
\end{tabular}

Values are medians among one-hundred rat and one-hundred guinea pig hearts.

Table 5. Summarized outcome of tests on different goodness-of-fit of relaxation fitting methods in onehundred rat and one-hundred guinea pig (GPig) hearts.

\begin{tabular}{|c|c|c|c|c|}
\hline \multirow{2}{*}{ Comparison } & \multirow{2}{*}{ Spec. } & \multirow{2}{*}{$\begin{array}{l}N^{*} \text { with } \\
p<0.05\end{array}$} & \multicolumn{2}{|c|}{ Agglutinated } \\
\hline & & & $Z$ & $P$ \\
\hline \multirow[t]{2}{*}{ S.1) Stdl3 vs. StdIL } & Rat & 44 & 8.1 & $<10^{-11}$ \\
\hline & GPig & 44 & 4.0 & $<2.8 \times 10^{-5}$ \\
\hline \multirow[t]{2}{*}{ S.2) StdIL vs. Stdl4 } & Rat & 65 & 39 & $<10^{-11}$ \\
\hline & GPig & 72 & 43 & $<10^{-11}$ \\
\hline \multirow[t]{2}{*}{ S.3) Stdl3 vs. Stdl3h } & Rat & 64 & 42 & $<10^{-11}$ \\
\hline & GPig & 45 & 18 & $<10^{-11}$ \\
\hline \multirow[t]{2}{*}{ S.4) Stdl3h vs. Stdl4 } & Rat & 10 & -5.3 & $>0.5$ \\
\hline & GPig & 30 & 10 & $<10^{-11}$ \\
\hline \multirow[t]{2}{*}{ E.1) ExtC3 vs. ExtCL } & Rat & 43 & 13 & $<10^{-11}$ \\
\hline & GPig & 57 & 26 & $<10^{-11}$ \\
\hline \multirow[t]{2}{*}{ E.2) ExtCL vs. ExtC4 } & Rat & 57 & 25 & $<10^{-11}$ \\
\hline & GPig & 71 & 38 & $<10^{-11}$ \\
\hline \multirow[t]{2}{*}{ E.3) ExtC3 vs. ExtC3h } & Rat & 58 & 27 & $<10^{-11}$ \\
\hline & GPig & 61 & 31 & $<10^{-11}$ \\
\hline \multirow{2}{*}{ E.4) ExtC3h vs. ExtC4 } & 4 Rat & 21 & 7.0 & $<10^{-11}$ \\
\hline & GPig & 30 & 20 & $<10^{-11}$ \\
\hline
\end{tabular}

* Number of experiments (also percentages) with $p<0.05$

Table 5 prove that the goodness-of-fit obtained from the four-dimensional exponentials StdI4 and ExtC4 is superior to the three-dimensional exponentials StdI3 and $\mathrm{ExtC} 3$, respectively. This result is directly interpretable as a test on $b_{\tau}=0$ against alternative $b_{\tau} \neq 0$ because the compared models differ only in that parame- 
Stdl
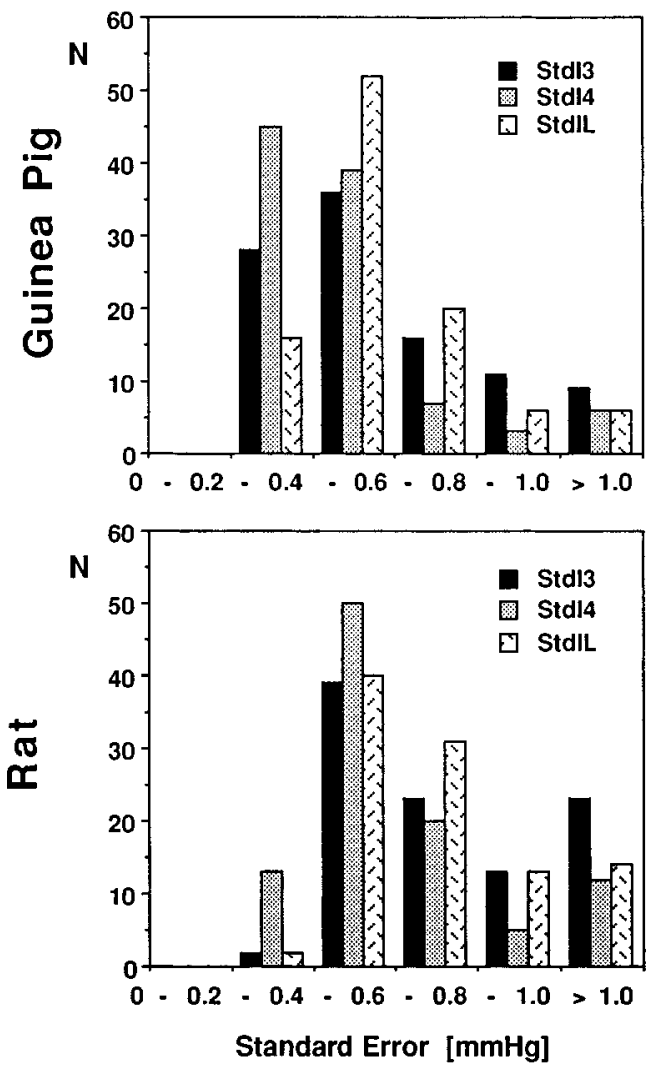

ExtC
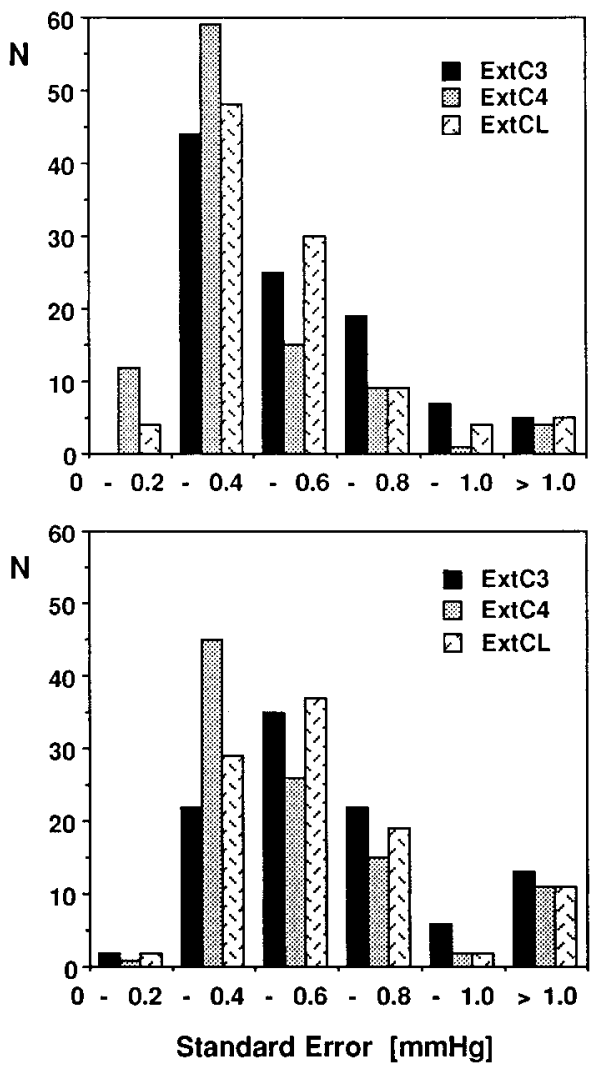

Fig. 4. Distribution of standard errors of regression determined from $N=100$ guinea pig and $N=100$ rat hearts. Standard errors up to $1 \mathrm{mmHg}$ are counted in five bins, each with $0.2 \mathrm{mmHg}$ width; cases with higher regression errors are collected in the last bin. See Table 1 for abbreviations. The central subinterval (ExtC) is reasonably better fitted by all models as compared to Stdl. The four-dimensional exponential fit according to Eq. 2 presents with least error sums. The pressure fall of guinea pig hearts always fits better (all models).

ter. The agglutinated test therefore proves that constancy of $\tau$ during relaxation (i.e., $b_{\tau}=0$ ) must not be assumed a priori.

Exceeding the level of significance is not difficult in the present analysis because pooling of all isovolumic pressure fall periods from the respective four-second LVP data sample provides a high degree of freedom. Comparing the distributions of the standard errors of regression, shown in Fig. 4, is therefore more instructive. The logistic model (Eq. 3) is slightly better than the three-parametric exponential (Eq. 1). The fourparametric exponential (Eq. 2) is optimal among the three methods compared in Fig. 4. The central interval ExtC tallies better with the regression models than the standard interval StdI does.

\section{DISCUSSION}

\section{Methodical considerations}

The present study is based on time series regression. Some mathematical models, describing isovolumic pressure fall, must therefore be proposed first.
The number of parameters of such a model is limited; more than four to six parameters cannot be reasonably estimated from the empirical data. Formulating a model in terms of myocellular properties, ventricular anatomy and geometry would lead to a multitude of unknown parameters, rendering this arrangement unfeasible. Fortunately, net effects describable by a few parameters usually emerge from such a multitude of interrelated factors and justify pressure fall models with a limited number of parameters.

This study compares models with three and four regression parameters. Increasing the number of parameters gives more flexibility to the fitting procedure and therefore causes the sum of the squared regression errors to decrease. Such effect is not mentioned as an empirical justification to adopt the more complicated model because the regression analysis primarily does not aim to improve fitting accuracy but intends to extract empirical information which is interpretable in terms of ventricular function. The purely mathematical improvement of fit is therefore eliminated by adjusting the degrees of freedom in calculating the $F$ 
statistic, Eq. 4 [16]. The squared error sum of the model that contains fewer parameters is divided by a higher degree of freedom than the error sum of the model with more parameters.

\section{Comparison of regression models}

The relaxation time constant $\tau$ is sometimes understood as being a purely empirical index [11]. However, knowledge about the physical meaning of the fitting models is necessary to interpret the results and is provided by the concomitant differential laws (see APPENDIX A). All three models this paper deals with propose that $\mathrm{p}(t)-p_{\infty}$, the actual intraventricular pressure above its asymptote, is a main driving force for its own decrease. This is quite reasonable: Suggesting for simplicity that all contractile force is vanishing suddenly at $t=0$, then the high intraventricular pressure, $p(0)$, will accelerate the viscoelastic cardiac wall and cause a change of ventricular shape making the ventricle capable of bearing the same volume at lower pressure. The pressure asymptote $\left(p_{\infty}\right)$ is a parameter extrapolated from the viscoelastic wall properties present during the selected isovolumic relaxation phase. $p_{\infty}$ should therefore not be expected to be equal to an empirical pressure asymptote measured in isovolumically beating hearts.

Equation 1 assumes proportionality between $\mathrm{p}(t)-$ $p_{\infty}$ and pressure fall velocity at each $t$. Equation 2 allows the time "constant" to become a linear function of $t$ during the pressure fall. The logistic model (Eq. 3 ) assumes that the initial time constant at $t=0$ is two times the asymptotic time constant $\tau_{\infty}$ (APPENDIX A). Matsubara et al. [13] have shown that the logistic model gives significantly less regression error than the three-parametric exponential in dog hearts. This has motivated the design of pairwise unilateral comparisons in the present study, and is approved by the present results in rat and guinea pig hearts. The better fit of the logistic model, compared with the three-parametric exponential, is in accordance with the finding of a negative slope of $\tau$ in the four-parametric exponential fit.

The present study has shown that Eq. 2 attains significantly less regression errors, compared with Eqs. 1 and 3, not explained just by introducing a fourth parameter. Equation 2 is also preferable if the information supplied by $b_{\tau}$ is not needed. In this case, $\tau$ should be taken from the center of the respective interval, $\tau=\tau^{*}=\tau_{0}+b_{\tau} t^{*}$, where $t^{*}$ is half the length of the chosen relaxation interval.

\section{Effect of interval tripartition}

The second newly introduced method to improve the goodness-of-fit is data-dependent interval tripartition. This method was originally performed by using the well-established three-dimensional model Eq. 1 [12]. However, further experience has shown that Eq. 1 fails to calculate a proper partition in about $15 \%$ of the pressure fall intervals. The reason was that this model supposes $\tau$ to be constant. Numerical instabilities occur while fitting the early and the terminal pressure data because estimated $\tau$ is far from being constant at these phases (see Fig. 1). The four-dimensional model (Eq. 2) is able to manage such changes of $\tau$ by allotting appropriate values to $b_{\tau}$. Tripartitions of pressure fall are therefore calculated only by the use of Eq. 2. This interval partition must not be confused with the three consecutive phases of the whole pressure fall, beginning at the onset of LVP fall with an "accelerative" phase, followed by an intermediate phase started after min $\mathrm{LVdP} / \mathrm{d} t$, and a terminal phase [3].

The parameters estimated from the early and late parts of ExtI are meaningless. The tripartition method yields unreasonably high $\tau$ values for the first and especially for the third subintervals, indicating that the exponential model (Eq. 2) cannot be properly applied to the pressure fall in the neighborhood of $\min \mathrm{LVdP} /$ $\mathrm{d} t$ and of re-encountered end-diastolic pressure. The reasons are slightly different in both cases, but they have a mathematical basis among them. It should first be emphasized that high $\tau$ values do not indicate "slow pressure fall" but only indicate "poor exponentiality" of pressure fall. This means that a straight line is better suited to fit the pressure data of such subintervals.

High $\tau$ values are estimated from the first subinterval of ExtI because the empirical pressure curve has an inflection point at $t=0$ (by definition of $\min \mathrm{LVdP} /$ $\mathrm{d} t$ ) and is therefore concave downward if $t<0$ and concave upward if $t>0$. On the other hand, Eq. 1 describes a function which is concave upward at every $t$ (supposing $\tau>0$ ). In consequence, the numerical fitting process balances between these incompatible demands by choosing a very high $\tau$, which renders Eq. 1 into an almost linear function. For example, suggest a realistic heart beat with $\operatorname{LVP}(0)=p_{0}=50 \mathrm{mmHg}$ and $\operatorname{LVdP}(0) / \mathrm{d} t=-\min \mathrm{LVdP} / \mathrm{d} t=-2,000 \mathrm{mmHg} \mathrm{s}^{-1}$. Estimating $\tau=500 \mathrm{~ms}$ and $p_{\infty}=-1,000 \mathrm{mmHg}$ is then almost equivalent to the linear model $\mathrm{p}(t)=p_{0}-$ $2 \mathrm{mmHg} \mathrm{ms}^{-1} \cdot t$ in the range of $t$ between $\pm 10 \mathrm{~ms}$. This consideration also applies to Eq. 2. Extraordinarily high $\tau$ values ( $>200 \mathrm{~ms}$ at control) were also found by Grocott-Mason et al. [17] in the pressure fall before $\min \mathrm{LVdP} / \mathrm{d} t$ in ejecting guinea pig hearts.

The meaningless high $\tau$ estimates from the last 
subinterval of ExtI are caused by numerical error. The late pressure values are always small, and the pressure fall itself is shallow. Therefore, measuremental noise and round-off errors due to A/D conversion falsify the data severely. The regression procedure mainly balances that random errors instead of the exponential pressure fall. This again yields an almost linear regression function (i.e., very high $\tau_{0}$ in Eq. 2) instead of an exponential.

In contrast, the exponential model (Eq. 2) has an excellent fit to the central portion (ExtC) of isovolumic pressure fall with median standard regression errors of 0.40 (rats) and $0.32 \mathrm{mmHg}$ (guinea pigs) (see Table 3); this is about one-half percent of the maximum systolic pressure. More reliable results, not attributable to the reduced data length, can therefore be obtained by discarding early and late pressure values by data-dependent optimal interval partition. By doing so, one should be aware that the disadvantage of fitting early and late isovolumic pressure data does not exclude that possibly important physiologic effects may be discarded. However, obtaining such information is on the limits of the discussed methods.

\section{Early and late isovolumic relaxation}

The significant improvement of regression using Eq. 2 leads to the aim of the present study, to test the hypothesis that $\tau$ may be considered to vary from early to late isovolumic relaxation rather than being constant. Combined comparisons S.1+S.2 and E. $1+$ E. 2 have proved that such variation really exists; in most cases, $\tau$ decreased during isovolumic relaxation $\left(b_{\tau}<0\right)$. Halfwise comparisons, StdI $3 \mathrm{~h}$ and ExtC3h, have also approved a smaller late $\tau$. This is in accordance with the work of Rousseau et al. [4], performing a piecewise semilogarithmic fit of the first and second half of pressure data separately. However, that method has fixed a zero asymptote and therefore does not allow real acceleration of relaxation and artificial effects introduced by improperly fixed asymptote to be discerned. Overcoming this objection, Raff and Glantz [7] have also noticed a slight time-related decrease of $\tau$ in canine hearts if the three-parametric model (Eq. 1) was applied to the first and last half of relaxation separately.

A diminishing $\tau$ is already taken into account by the logistic model Eq. 3 (see APPENDIX A), but not with sufficient flexibility. Introducing a fourth parameter was statistically proved to be worth the cost (i.e., $b_{\tau}$ in Eq. 2 bears actual information instead of random noise). This is a necessary but not sufficient prerequisite to consider the fourth parameter as valuable to describe isovolumic relaxation. The (patho-) physiological role of $b_{\tau}$ therefore needs further experience. In order to find a physiological example demonstrating the usefulness or necessity of the four-dimensional description of isovolumic relaxation, one has to search for hemodynamic parameters that influence the factor $b_{\tau}$. The influence of combined changes of left ventricular end-diastolic and maximum pressure and of heart rate on $\tau$ (Eq. 1) and $\tau_{0}, b_{\tau}$ (Eq. 2) was recently studied [18]. It was shown that $\tau$, but not the central time constant $\tau^{*}$ estimated from Eq. 2, significantly increases with beat interval length. Pacing experiments are therefore expected to demonstrate qualitative differences between the relaxation models. The data displayed in Fig. 5 prove that none of the threedimensional models is sufficient to describe the lusitropic effect of pacing properly, in contrast to the four-dimensional model.

The results from Fig. 5 may be explained by changes of intracellular $\mathrm{Ca}^{2+}$ handling. Myofibrillar relaxation depends on the speed by which $\mathrm{Ca}^{2+}$ is released from the low-affinity site of the troponin C subunit $[19,20]$; this is a "multi compartment process" involving free cytosolic calcium, the $\mathrm{Ca}^{2+}$ uptake by sarcoplasmatic reticulum, and $\mathrm{Ca}^{2+}$ efflux through $\mathrm{Na}^{+}-\mathrm{Ca}^{2+}$-exchanger [21]. Removing $\mathrm{Ca}^{2+}$ from the contractile proteins is not completed at the beginning isovolumic pressure fall under certain conditions [1, 20]. Ongoing $\mathrm{Ca}^{2+}$ removal is expected to cause $\tau$ to further decrease (as in Fig. 5 at normal BI) because the myocardium becomes less viscous in its relaxed state $[22,23]$. At short BI, on the other hand, the increasing number of action potentials per time unit increases the cytoplasmatic $\mathrm{Ca}^{2+}$ load, which in turn enhances the activity of sarcoplasmatic calcium ATPase [24]. The $\mathrm{Ca}^{2+}$ entering the cytoplasma during the individual action potential is therefore removed more rapidly at high heart rate. Figure 5 suggests that, at high heart rate, the myocardium is fully relaxed before isovolumic condition begins because the initial time constant is already minimal and does not further decrease $\left(b_{\tau} \approx 0\right)$.

Generally, $b_{\tau}$ indicates distinct physiological changes in the transition from partially contracted to fully relaxed myocardial wall. Asynchrony of relaxation among the multitude of myocardial filaments is therefore another possible source of impaired early relaxation. Even small strips of myocardial muscle present with considerable asynchrony [25, 26], and this is also an important property of the intact ventricle [3, 25]. Considerable partial contraction is still present in early isovolumic pressure fall [27] albeit relaxation begins before peak negative pressure fall velocity occurs. The well-known higher viscosity of contracted 


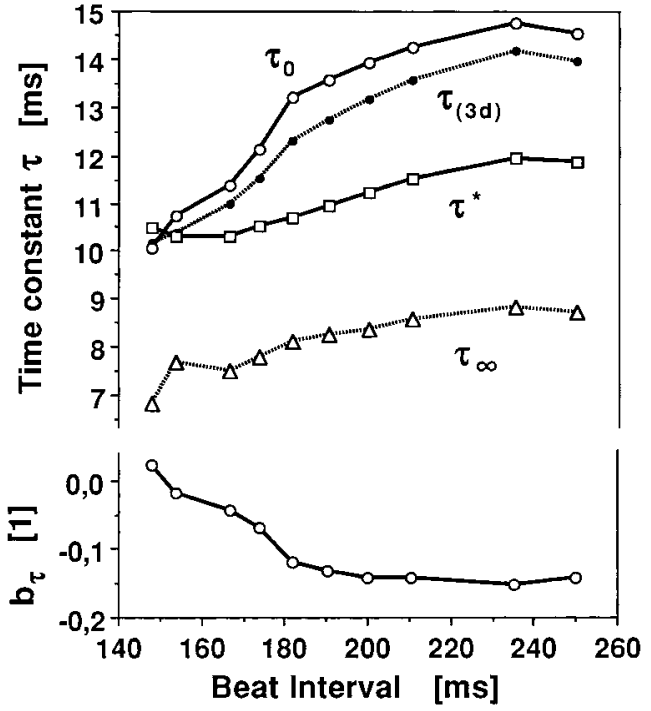

Fig. 5. Time constants of isovolumic pressure fall (Stdl) of an isolated working rat heart during right atrial electrical stimulation with increasing frequency. Average aortic pressure $(75 \mathrm{mmHg})$, cardiac flow $(40.2 \mathrm{ml}$ $\left.\mathrm{min}^{-1}\right)$, and temperature $\left(36.9^{\circ} \mathrm{C}\right)$ were held constant. Electrical stimulation started at a frequency slightly above the intrinsic sinus node frequency (right hand side of the panel) and was accelerated stepwise to the shortest beat interval (BI) tolerated by the heart. The exponential time constant $\tau_{(3 \mathrm{~d})}$, estimated by Eq. 1, decreases considerably with BI length indicating accelerated relaxation at high frequency. In contrast, the asymptotic ("late") logistic time constant $\tau_{\infty}$ (Eq. 3) suggests that the lusitropic effect of BI length is only small. The four-dimensional exponential model (Eq. 2) unifies these observations: Only the initial time constant, $\tau_{0}$, increases at long Bls. The central time constant $\tau^{\star}$ is much more $\mathrm{Bl}$-independent. The $b_{\tau}$ curve shows that isovolumic relaxation accelerates during isovolumic pressure fall at normal but not at very short BI. Equation 1 fails to detect acceleration at normal $\mathrm{BI}$. Equation 3 is unsuitable to describe the pressure fall at short $\mathrm{BI}$ (with $b_{\tau} \approx 0$ ) properly because it always suggests that the initial time constant is two times $\tau_{\infty}$.-Calculating the curves from the central subinterval ExtC instead from Stdl yields a less marked change of $\tau_{(3 \mathrm{~d})}$ and $\tau_{0}$ with $\mathrm{BI}$ length. This was to be expected because the most $\mathrm{BI}$-sensitive $\tau_{0}$ is defined as initial time constant; $\tau_{0}$ is therefore strongly influenced by the very first values of the isovolumic pressure fall that are absent from ExtC.

myocardium $[22,23]$ may therefore be responsible for higher $\tau$ (i.e., impaired early relaxation). Artificial nonuniformity has been produced by isoproterenol injection in mid-left anterior descending coronary artery $[8,28]$ and has elevated the relaxation time constant. Asynchrony is also aggravated in pathologic conditions [2].

Coronary vascular engorgement [29] may also contribute to decreasing $\tau$ during isovolumic relaxation. However, such mechanical effects must be separated from the effect of ameliorated oxygen supply in late diastole due to higher coronary flow through the relaxed myocardium. Reducing cardiac oxygen supply (below $100 \mu 1 \mathrm{~min}^{-1} \mathrm{~g}^{-1}$ ) at a constant perfusion pressure has been demonstrated to prolong $\tau$ in guinea pig hearts [5], pointing to predominance of oxygen supply over simple pressure effects. Corresponding results were obtained from patients during coronary occlusion [6]. Therefore, oxygen deficiency may explain the regional nonuniformity of relaxation in dilated cardiomyopathy, for which the cause has been suggested as being unclear [2].

Whereas asynchrony and improved oxygen supply may explain the acceleration of relaxation found in the present study, the bending of some elastic wall elements late in relaxation and the vanishing intrinsic restoring force of contracted muscle fibers [30] may contribute, on the other hand, to an increasing $\tau$; however, most hearts in the present study do not reveal any such effects. One may speculate about the minority of hearts which presented with lower early and higher late isovolumic relaxation constants. Myocardial relaxation may have already been more complete in these hearts before isovolumic conditions were reached, or contraction may have been insufficient.

\section{Interbeat variability of the parameters}

All regression methods described here were also found to be applicable to single beats (instead to pooled data from several consecutive beats) without numerical instability. The isolated hearts of this study presented with quite small interbeat variability of the parameters (Table 4). High coefficients of variability are seen in $p_{\infty}$ and $b_{\tau}$; it must be considered that the values of both parameters are small and can freely cross zero. Additionally, some variability of $p_{\infty}$ may be attributable to the Frank-Starling mechanism: $p_{\infty}$ depends on the residual volume [22], which is greater after a weak contraction. The resulting greater residual volume in turn leads to a higher diastolic filling enhancing the next contraction, which therefore yields a smaller residual volume. Most parameters were more variable in method ExtC4, the four-dimensional fit of the central subinterval of the extended pressure fall interval; this effect is due to the individual datadependent determination of the subinterval which introduces additional variability.

Martin et al. [10] have found differences of $1.8 \mathrm{~ms}$ for $\tau$ and $3 \mathrm{mmHg}$ for $p_{\infty}$ in runs of four consecutive beats in anesthetized dogs. Simari et al. [6] have reported coefficients of variations obtained from humans; variation of $\tau$ (exponential fit) was $23 \%$ and that of $p_{\infty}$ was $69 \%$. These greater variabilities, compared to isolated rat and guinea pig hearts, may be at- 
tributed to autonomous nervous activity. Analyzing the isovolumic pressure fall beat by beat may therefore be necessary under such circumstances.

\section{Limitations and conclusion}

This study is confined to isolated hearts of two species working under "medium" hemodynamic conditions. The present results may differ from those under other working conditions which may change the pressure curve or relaxation time. However, the "basic" regression formula (Eq. 1) has been demonstrated to be reliable in assessing the relaxation time constants from rat and guinea pig hearts under various conditions, including high frequent pacing and hypothermia, with remarkably changed pressure pulse form [9].

Which method should be practically applied? The three-parametric exponential fit Eq. 1 of the standard interval StdI yields regression errors less than 3\% of the inital pressure $p_{0}$ (less than $1 \%$ of maximum LVP). This is already a very good fit of a biological time series. Using a fixed zero asymptote [10] yielded significantly greater regression error [9]; three regression parameters therefore seem to be necessary to perform a proper fit. Figure 4 reveals only a slight improvement of regression by the logistic model (Eq. 3) as compared with Eq. 1. Comparing SERs in Table 3 shows that introducing the fourth parameter $b_{\tau}$ (Eq. 2) decreases the regression error by about $20 \%$. A statistical test proved that $b_{\tau}$ contains significant information. Another $10 \%$ reduction of SER is attainable by selecting a central interval (ExtC) of isovolumic relaxation by data-dependent interval partition using Eq. 2 . This method requires considerable computational expense. However, Fig. 1 demonstrates that the removal of early and late pressure data from the regression is desirable. The influence of poorly fitting parts on the relaxation parameters was not investigated in detail by the present study. Some pathologic or hemodynamic conditions (e.g., oxygen deficiency or high heart rate, as in Fig. 5) may aggravate the differences between parameters taken from StdI and ExtC, respectively.

To summarize: (1) The pressure fall during isovolumic relaxation of the left ventricle is fairly well described by a three-parametric monoexponential regression function. The goodness-of-fit is significantly enhanced by assuming the time constant as a linear time-variant function, thus introducing a fourth regression parameter, the slope of the time constant. (2) Deletion of the poorly fitting early and late isovolumic pressure fall by optimal data-dependent interval partition further decreases the regression error remarkably, and is therefore advantageous, especially if pooled pressure fall data of multiple consecutive beats are available. (3) Four-dimensional exponential regression is applicable to single-beat data in rat and guinea pig hearts also combined with discarding of early and late pressure fall data. (4) The relaxation constant is higher in the early isovolumic phase than in the later phase under medium hemodynamic conditions. This is probably due to a still partially contracted myocardium.

\section{REFERENCES}

1. Smith VE, Weisfeldt ML, and Katz AM: Relaxation and diastolic properties of the heart. In: The Heart and Cardiovascular System, ed. Fozzard HA, Raven Press, New York, pp 803-817, 1986

2. Hayashida W, Kumada T, Kohno F, Noda M, Ishikawa $\mathrm{N}$, Kambayashi M, and Kawai C: Left ventricular relaxation in dilated cardiomyopathy: relation to loading conditions and regional nonuniformity. J Am Coll Cardiol 20: 1082-1091, 1992

3. Leite-Moreira AF and Gillebert TC: Nonuniform course of left ventricular pressure fall and its regulation by load and contractile state. Circulation 90: 2481-2491, 1994

4. Rousseau MF, Veriter C, Detry JM, Brasseur L, and Pouleur $\mathrm{H}$ : Impaired early left ventricular relaxation in coronary artery disease: effects of intracoronary nifedipine. Circulation 62: 764-772, 1980

5. Schäfer S, Schlack W, Kelm M, Deussen A, and Strauer $\mathrm{BE}$ : Characterisation of left ventricular relaxation in the isolated guinea pig heart. Res Exp Med 196: 261-273, 1996

6. Simari B, Bell MR, Schwartz RS, Nishimura RA, and Holmes DR Jr: Ventricular relaxation and myocardial ischemia: a comparison of different models of tau during coronary angioplasty. Cathet Cardiovasc Diagn 25: 278-284, 1992

7. Raff GL and Glantz SA: Volume loading slows left ventricular isovolumetric relaxation rate; evidence of loaddependent relaxation in the intact dog heart. Circ Res 48: 813-824, 1981

8. Lew WY and Rasmussen CL: Influence of nonuniformity on rate of left ventricular pressure fall in the dog. Am J Physiol 256: H222-H232, 1989

9. Langer SF and Schmidt HD: Different left ventricular relaxation parameters in isolated working rat and guinea pig hearts. Influence of preload, afterload, temperature, and isoprenaline. Int $\mathrm{J}$ Cardiac Imaging 14: 229-240, 1998

10. Martin G, Gimeno JV, Cosin J, and Guillem I: Time constant of isovolumic pressure fall: new numerical approaches and significance. Am J Physiol 247: H283-H294, 1984

11. Thompson DS, Waldron CB, Coltart DJ, Jenkins BS, and Webb-Peploe MM: Estimation of time constant of left ventricular relaxation. Br Heart J 49: 250-258, 1983

12. Langer SF: Data-dependent interval partition of naturally ordered individuals by complete cluster analysis in epidemiological and cardiac data processing. Statist Med 16: 1617-1628, 1997 
13. Matsubara H, Takaki M, Yasuhara S, Araki J, and Suga $\mathrm{H}$ : Logistic time constant of isovolumic relaxation pressure-time curve in the canine left ventricle. Better alternative to exponential time constant. Circulation 92: 2318-2326, 1995

14. Langer SF: Efficient exponential regression with exact fiducial limits to fit cardiac pressure data. Comput Methods Programs Biomed 53: 57-64, 1997

15. Press WH, Flannery P, Teukolsky SA, and Vetterling WT: Numerical Recipes in Pascal. The Art of Scientific Computing, Cambridge Univ Press, Cambridge, 1989

16. Motulsky $\mathrm{HJ}$ and Ransnas LA: Fitting curves to data using nonlinear regression: a practical and nonmathematical review. FASEB J 1: 365-374, 1987

17. Grocott-Mason R, Anning P, Evans $H$, Lewis MJ, and Shah AM: Modulation of left ventricular relaxation in isolated ejecting heart by endogenous nitric oxide. Am J Physiol 267: H1804-H1813, 1994

18. Langer SF: Multivariate regression analysis of the influence of aortic pressure, end-diastolic pressure, and heart rate on left ventricular relaxation in isolated ejecting rat and guinea pig hearts. Res Exp Med 199: 153-166, 1999

19. Tobias AH, Slinker BK, Kirkpatrick RD, and Campbell KB: Mechanical determinants of left ventricular relaxation in isovolumically beating hearts. Am $\mathrm{J}$ Physiol 268: H170-H177, 1995

20. Peterson JN, Hunter WC, and Berman MR: Estimated time course of $\mathrm{Ca}^{2+}$ bound to troponin $\mathrm{C}$ during relaxation in isolated cardiac muscle. Am J Physiol 260: $\mathrm{H} 1013-\mathrm{H} 1024,1991$

21. Vittone L, Mundiña-Weilenmann C, Mattiazzi A, and Cingolani $\mathrm{H}$ : Physiologic and pharmacologic factors that affect myocardial relaxation. J Pharmacol Toxicol Meth 32: 7-18, 1994

22. Brecher GA: Critical review of recent work on ventricular diastolic suction. Circ Res 6: 554-566, 1958

23. Templeton GH and Nardizzi LR: Elastic and viscous stiffness of the canine left ventricle. J Appl Physiol 36: 123-127, 1974

24. James P, Inui M, Tada M, Chiesi M, and Carafoli E: Nature and site of phospholamban regulation of the $\mathrm{Ca}^{2+}$ pump of sarcoplasmatic reticulum. Nature 342: 90-92, 1989

25. Blaustein AS and Gaasch WH: Myocardial relaxation. VI. Effects of $\beta$-adrenergic tone and synchrony on LV relaxation rate. Am J Physiol 244: H417-H422, 1983

26. Brutsaert DL: Nonuniformity: a physiologic modulator of contraction and relaxation of the normal heart. $\mathrm{J} A \mathrm{~m}$ Coll Cardiol 9: 341-348, 1987

27. Nikolic SD, Tamura K, Tamura T, Dahm M, Frater RW, and Yellin EL: Diastolic viscous properties of the intact canine left ventricle. Circ Res 67: 352-359, 1990

28. Gillebert TC and Lew WY: Nonuniformity and volume loading independently influence isovolumic relaxation rates. Am J Physiol 257: H1927-H1935, 1989

29. Salisbury PF, Cross CE, and Rieben PA: Influence of coronary artery pressure upon myocardial elasticity. Circ Res 8: 794-800, 1960

30. Parsons $C$ and Porter KR: Muscle relaxation: evidence for an intrafibrillar restoring force in vertebrate striated muscle. Science 153: 426-427, 1966

\section{APPENDIX A}

\section{Differential law of isovolumic pressure fall}

The general differential law fulfilled by the abovementioned regression functions fitting the isovolumic pressure fall is;

$$
\frac{\mathrm{d}\left[\mathrm{p}(t)-p_{\infty}\right]}{\mathrm{d} t}=\frac{\mathrm{dp}(t)}{\mathrm{d} t}=\frac{-1}{\tau(t)}\left[\mathrm{p}(t)-p_{\infty}\right],
$$

stating that, conveyed by $\tau(t)^{-1}$, the pressure decay (notice $-\tau(t)^{-1}<0$ ) velocity depends on the distance of the actual pressure $\mathrm{p}(t)$ from asymptotic pressure $p_{\infty}$.

In the three-parametric function (Eq. 1), known as the aperiodic limiting case of oscillation, $\tau(t)=\tau$ is a time constant.

In the four-parametric fitting function (given in Eq. 2), $\tau(t)=\left(\tau_{0}+b_{\tau} t\right)^{2} \tau_{0}{ }^{-1}=\tau_{0}+b_{\tau}\left(2+b_{\tau} \tau_{0}{ }^{-1} t\right) t$ holds. $\tau_{0}=\tau(0)$ is the initial time constant. $\tau(t)$ is not a linear function, but the deviation from linearity is rather small in the apparent range of the parameters. Linearity of the denominator $\tau_{0}+b_{\tau} t$ in Eq. 2 was preferred to linearity of $\tau(t)$ in the differential law because Eq. 2 is a transparent extension of the well-established exponential pressure fall representation (Eq. 1).

The logistic function, Eq. 3, yields

$$
\tau(t)=\tau_{\infty}\left(1-\frac{1}{2} \cdot \frac{\mathrm{p}(t)-p_{\infty}}{p_{0}-p_{\infty}}\right)^{-1}=\tau_{\infty}\left(1+\exp \frac{-t}{\tau_{\infty}}\right) .
$$

It is $\tau_{\infty}=\lim _{t \rightarrow \infty} \tau(t)$, and the initial time constant is $\tau_{0}=\tau(0)=2 \tau_{\infty} . \tau$ decreases during the fall of $\mathrm{p}(t)-p_{\infty}$. The logistic function was originally introduced by Verhulst to describe the growth of a population, where high levels of population retard their further increase. This means, in the present case of pressure fall function, that high pressure slightly hampers its own decrease. It seems reasonable to take the initially high intraventricular pressure as a measure of wall stress caused by residually contracted myofilaments. Higher $\tau$ at this condition reflects higher viscosity of the contracted myocardium. However, the assumtion that initial $\tau_{0}$ is doubled to as high as the asymptotic $\tau_{\infty}$ is a restriction which is not justified theoretically or empirically.

\section{APPENDIX B}

\section{Search for global error minima}

The simplex algorithm applied in the present study for least-squares parameter estimation performs only local error minimizing, beginning with user supplied 
initial estimates. Improper start values may cause the algorithm to become trapped in some local minimum of the empirical squared-error curve which is generated artificially by round-off errors [16]. This phenomenon was likewise found in fitting the four-parametric model (Eq. 2). In some cases, especially in slowly decreasing late pressure data, almost the same error sums could be obtained by very different sets of parameters. It was therefore necessary to search for global minima before applying local optimization. The following procedure was developed in hearts not included in the present study.

For fitting pressure intervals StdI and ExtC, ranges where to search for error minimizing parameters were initially set as follows: $p_{0}=10$ to $90 \mathrm{mmHg}, p_{\infty}=-80$ to $50 \mathrm{mmHg}, \tau_{0}=12$ to $50 \mathrm{~ms}$, and $b_{\tau}=-1$ to 1 . Eight values were equidistantly chosen from each range, thus forming a four-dimensional grid of $8^{4}=4,096$ points. Squared error sums were calculated for each set of parameters, retaining the best twenty sets. A neighborhood around each of these best parameters was checked; the range of values was set to one-fourth of the respective initial range, and the described procedure was carried out again. Three recursive repetitions of this refinement, always retaining the twenty best sets of parameters encountered, yielded optimal results; neither less nor more recursions were preferable. Four input sets (the simplex method neeeds five sets of initial values to minimize a four-parametric function) were formed from these twenty parameter sets and subjected to simplex optimization. The computational effort of that global parameter search is considerably high, about $90 \%$ of total computing time. The method must therefore be considered as preliminary; further enhancement is possible and desired. 